\title{
AN ALTERNATIVE PROOF OF A THEOREM ON \\ THE LEBESGUE INTEGRAL
}

\author{
by B. D. JOSEPHSON
}

The theorem concerned is the following:

if $f$ is continuous in $[a, b]$, and $f^{\prime}$ exists and is finite except at an enumerable set of points and Lebesgue integrable in $[a, b]$, then

$$
\int_{a}^{b} f^{\prime}=f(b)-f(a)
$$

Proof. We assume the following theorems:

(1) the above formula is true if $f$ is absolutely continuous (a.c.) in $[a, b]$;

(2) an indefinite integral is a.c.

We first prove the following lemma:

if $f^{\prime}$ is integrable, and $f$ continuous but not a.c. in $[a, b]$, then given $K>0$, $l>0$, there exist two disjoint subintervals $\left[a^{\prime}, b^{\prime}\right]$ of $[a, b]$ such that

$$
b^{\prime}-a^{\prime}<l,\left|f\left(b^{\prime}\right)-f\left(a^{\prime}\right)\right|>K\left(b^{\prime}-a^{\prime}\right),
$$

and $f$ is not a.c. in $\left[a^{\prime}, b^{\prime}\right]$.

Proof. Since $f$ is not a.c. in $[a, b], \exists \varepsilon>0$ such that given $\delta>0$, there is a finite set of non-overlapping intervals $\left[a_{r}, b_{r}\right]$ such that

$$
\Sigma\left(b_{r}-a_{r}\right)<\delta, \quad \Sigma\left|f\left(b_{r}\right)-f\left(a_{r}\right)\right|>\varepsilon .
$$

By (2), $F(x) \equiv \int_{a}^{x}\left|f^{\prime}\right|$ is a.c. Hence $\exists \delta_{1}>0$ such that if $\left\{\left[a_{r}, b_{r}\right]\right\}$ is a finite set of non-overlapping intervals and $\Sigma\left(b_{r}-a_{r}\right)<\delta_{1}$, then

$$
\Sigma\left|F\left(b_{r}\right)-F\left(a_{r}\right)\right|<\frac{1}{8} \varepsilon_{\text {. }}
$$

By uniform continuity, $\exists \delta_{2}>0$ such that if $\left|b_{r}-a_{r}\right|<\delta_{2}$, then

$$
\left|f\left(b_{r}\right)-f\left(a_{r}\right)\right|<\frac{1}{4} \varepsilon \text {. }
$$

Now take $\delta=\min \left(\delta_{1}, \delta_{2}, l, \varepsilon / 8 K\right)$, and choose intervals $\left[a_{r}, b_{r}\right]$ to satisfy (3). The sum $\Sigma\left|f\left(b_{r}\right)-f\left(a_{r}\right)\right|$ may be divided into three parts, by putting $\left|f\left(b_{r}\right)-f\left(a_{r}\right)\right|$ into

$\Sigma_{1}$ if $f$ is a.c. in $\left[a_{r}, b_{r}\right]$,

$\Sigma_{2}$ if $f$ is not a.c. in $\left[a_{r}, b_{r}\right]$ and $\left|f\left(b_{r}\right)-f\left(a_{r}\right)\right| \leqq K\left(b_{r}-a_{r}\right)$,

$\Sigma_{3}$ if $f$ is not a.c. in $\left[a_{r}, b_{r}\right]$ and $\left|f\left(b_{r}\right)-f\left(a_{r}\right)\right|>K\left(b_{r}-a_{r}\right)$.

Now if $f$ is a.c. in $\left[a_{r}, b_{r}\right]$, then by (1),

E.M.S. -

$$
\left|f\left(b_{r}\right)-f\left(a_{r}\right)\right|=\left|\int_{g_{r}}^{b_{r}} f^{\prime}\right| \leqq \int_{a_{r}}^{b_{r}}\left|f^{\prime}\right|=\left|F\left(b_{r}\right)-F\left(a_{r}\right)\right| .
$$


Hence $\quad \Sigma_{1}\left|f\left(b_{r}\right)-f\left(a_{r}\right)\right| \leqq \Sigma_{1}\left|F\left(b_{r}\right)-F\left(a_{r}\right)\right|<\frac{1}{8} \varepsilon$ by (4).

Also $\quad \Sigma_{2}\left|f\left(b_{r}\right)-f\left(a_{r}\right)\right| \leqq \Sigma_{2}\left\{K\left(b_{r}-a_{r}\right)\right\}<K \delta \leqq \frac{1}{8} \varepsilon$.

Hence by (3), $\Sigma_{3}\left|f\left(b_{r}\right)-f\left(a_{r}\right)\right|>\frac{3}{4} \varepsilon$, and so from (5) it follows that $\Sigma_{3}$ has at least three terms. Two of the corresponding intervals $\left[a_{r}, b_{r}\right]$ must be disjoint, and from the definition of $\Sigma_{3}$ satisfy the conditions of the lemma.

Now let $f$ be a function satisfying the hypothesis of the main theorem, and let $x_{1}, x_{2}, x_{3}, \ldots$ be the set of points at which $f^{\prime}$ does not exist. If the theorem does not hold, then by (1), $f$ is not a.c. in $[a, b]$. We now construct inductively a set of intervals $I_{r}=\left[a_{r}, b_{r}\right]$ with the following properties $(r \geqq 1)$ :

$$
b_{r}-a_{r}<1 / r,\left|f\left(b_{r}\right)-f\left(a_{r}\right)\right|>r\left(b_{r}-a_{r}\right), I_{r} \subset I_{r-1}, x_{r} \notin I_{r},
$$

and $f$ is not a.c. in $I_{r}$.

Let $I_{0}$ be $[a, b]$. If $I_{r-1}$ has been constructed, the lemma may be applied to obtain two disjoint subintervals such that $b^{\prime}-a^{\prime}<1 / r,\left|f\left(b^{\prime}\right)-f\left(a^{\prime}\right)\right|>r\left(b^{\prime}-a^{\prime}\right)$ and $f$ is not a.c. in $\left[a^{\prime}, b^{\prime}\right]$. At least one of these does not contain $x_{r}$. Take this to be $I_{r}$.

Then $a_{0} \leqq a_{1} \leqq a_{2} \leqq \ldots, b_{0} \geqq b_{1} \geqq b_{2} \geqq \ldots, 0<b_{r}-a_{r}<1 / r$, and so $\left\{a_{r}\right\},\left\{b_{r}\right\}$, tend to a common limit $x$, contained in each $I_{r}$, and hence different from each $x_{r}$, so that $f^{\prime}(x)$ exists and is finite.

Now $\frac{f\left(b_{r}\right)-f\left(a_{r}\right)}{b_{r}-a_{r}}$ lies between $\frac{f\left(b_{r}\right)-f(x)}{b_{r}-x}$ and $\frac{f\left(a_{r}\right)-f(x)}{a_{r}-x}$, each of which tends to $f^{\prime}(x)$ as $r \rightarrow \infty$. But $\left|\frac{f\left(b_{r}\right)-f\left(a_{r}\right)}{b_{r}-a_{r}}\right| \geqq r$, all $r$, and this contradiction proves the theorem.

Trinity CoLlege

CAMBRIDGE 